\title{
Comparison of metabolic abnormalities in patients with new-diagnostic polycystic ovary syndrome and with new-diagnostic Type 2 diabetes mellitus
}

\author{
Fang Fang, Minyu Gu, Lin Chen, Na Li, Xiaoying Ding, Yongde Peng* \\ Department of Endocrine and Metabolism, Shanghai First People’s Hospital Affiliated to Shanghai Jiao Tong University, Shanghai, \\ China; ${ }^{*}$ Corresponding Author: pyongde@yeah.net
}

Received 6 January 2014; revised 3 February 2014; accepted 10 February 2014

Copyright (C) 2014 Fang Fang et al. This is an open access article distributed under the Creative Commons Attribution License, which permits unrestricted use, distribution, and reproduction in any medium, provided the original work is properly cited. In accordance of the Creative Commons Attribution License all Copyrights (C) 2014 are reserved for SCIRP and the owner of the intellectual property Fang Fang et al. All Copyright (C) 2014 are guarded by law and by SCIRP as a guardian.

\section{ABSTRACT}

Background: Metabolic abnormalities have been widely studied in women with polycystic ovary syndrome (PCOS) and in patients with Type 2 diabetes mellitus (T2DM). However, there are few studies on the comparison of the metabolic disturbances between the two diseases although they have partly common pathogenesis. This study compares the metabolic parameters of PCOS and T2DM, and evaluates the association between metabolic parameters and gonadotropic hormones. Methods: Fifity newly diagnosed PCOS women and 56 newly diagnosed Type 2 diabetes women were enrolled in the study. The metabolic parameters including body mass index, waist to hip ratio, blood pressure, total cholesterol, triglycerides, high-density and low-density lipoprotein cholesterol, fasting and postprandial plasma glucose, fasting and postprandial plasma insulin, HOMA-IR index and uric acid, along with the gonadal and gonadotropic hormones were analyzed between the two groups. The lutein hormone (LH) responses to the GnRH stimulating test were compared between different glucose tolerance groups in PCOS women. The peak values of LH in the GnRH test are studied with metabolic parameters using Pearson correlation analysis. Results: PCOS patients have higher body weight index, waist to hip ratio, fasting and postprandial insulin, HOMA-IR and uric acid than T2DM patients. There are no significant differences in lipid metabolism excluding the age influence. On the other hand, T2DM patients have significantly higher systolic blood pressure and plasma glucose than those in PCOS patients. But there is less clinical significance in the level of plasma glucose. After GnRH injection in PCOS women, there are significant differences in $\mathrm{LH}$ reaction between the three groups with different glucose tolerance. Conclusion: Women with PCOS have more severe metabolic disturbance than women with T2DM except for systolic blood pressure. Insulin resistance affects the function of thalamus-pituitary-gonad axis.

\section{KEYWORDS}

PCOS; Type 2 Diabetes Mellitus; Metabolic Abnormalities

\section{BACKGROUND}

Polycystic ovary syndrome (PCOS) is a heterogeneous endocrine disorder in reproductive-aged women characterized by hyperandrogenism and chronic anovulation. It is associated with an increased risk for metabolic complications and women with PCOS tend to have more than two to four times metabolic disturbances compared to those without PCOS [1-3]. Hyperinsulinemia reflecting some degree of peripheral insulin resistance was well recognized in PCOS by the mid-1980s. It is now recognized that insulin resistance, which is recognized as a major risk factor for the development of Type 2 diabetes mellitus (T2DM), and hyperinsulinemia both play important roles in the pathogenesis of PCOS. Metabolic abnormalities have been widely studied in women with PCOS and in patients with T2DM, but none of these studies provide a comparison of the metabolic disturbances 
occurring in these two conditions. Therefore, we aim to compare the metabolic parameters in women with PCOS and with T2DM.

An oral glucose tolerance test (OGTT) and a gonadotropin releasing hormone $(\mathrm{GnRH})$ stimulation test were performed in all women with PCOS in the present study. The serum lutein hormone (LH) was elevated significantly after GnRH test. PCOS women are at substantially higher risk for impaired glucose tolerance (IGT) and T2DM. This poses the question of the presence of a relationship between the increased pituitary sensitivity and the glucose tolerance status. Hence, we also compare the $\mathrm{LH}$ reaction after $\mathrm{GnRH}$ stimulation test among three groups with different glucose tolerance status and explore the correlation between the peak value of $\mathrm{LH}$ and the metabolic parameters in PCOS women.

\section{METHODS}

\subsection{Patients Identification}

A total of 106 consecutive patients were included in the study. 50 women were diagnosed as PCOS and 56 other women suffered from T2DM. All patients included in the study were new-diagnostic subjects for avoiding the disturbances of duration and drugs.

The new-diagnostic PCOS subjects should present at least two of the following criteria after exclusion of other etiologies (e.g. congenital adrenal hyperplasia, androgen-secreting tumors and Cushing's syndrome): 1) oligoand/or anovulation (i.e. $\leq 8$ menstrual periods in a year or menstrual cycles more than 35 days in length); 2) clinical hyperandrogenism and/or biochemical hyperandrogenism; 3) polycystic ovaries (i.e. the presence of $\geq 12$ follicles in each ovary measuring $2-9 \mathrm{~mm}$ in diameter). They also should be diagnosed within one month and without any prior medical treatments.

The newly-diagnosed T2DM patients should meet the following criteria: 1) the 1997 ADA criteria; 2) diagnosed within one month; 3) without any prior medical treatments; 4) women.

\subsection{Study Protocols}

All subjects who were enrolled in the study underwent physical examination. Body mass index (BMI) and Waist to hip ratio (WHR) was calculated. Blood pressure was measured in the supine position and averaged over three measurements. Waist circumference was measured at the widest point between the lower border of the right costal margin and the superior point of the iliac crest.

OGTT was performed between $08 \mathrm{am}$ - 10 am after a 3-day 300 g carbohydrate diet and an overnight fast of 10 - $14 \mathrm{~h}$ in PCOS women. Blood was obtained for glucose determinations at $0 \mathrm{~h}$ (FPG) and $2 \mathrm{~h}$ (2hPG). Insulin levels were also determined in these samples (FINS,
2hINS). An additional blood sample was obtained at $0 \mathrm{~h}$ for triglycerides (TG), total cholesterol (CHOL), highdensity lipoprotein cholesterol (HDL-C), low-density lipoprotein cholesterol (LDL-C) and uric acid (UA).

GnRH stimulation test was performed at 08 am - 10 am on another day. An intravenous cannula was inserted and blood samples were collected for basal lutein hormone ( $\mathrm{LH})$, follicle stimulating hormone (FSH), total testosterone (T), prolactin (PRL) and estradiol (E2). Following administration of a standard dose of $100 \mathrm{mg}$ GnRH (Gonadorelin for injection, Anhui BBCA Pharmaceuticals Co., Ltd, China), blood samples for LH were obtained at the $15^{\text {th }}, 30^{\text {th }}, 60^{\text {th }}, 90^{\text {th }}$ and $120^{\text {th }}$ minutes.

We use revised homeostatic model assessment (HOMA, proposed by Matthews DR in 1998 [4]) to evaluate $\beta$-cell insulin resistance. The computer program was downloaded from the website of www.dtu.ox.ac.uk.

\subsection{Assays}

LH, FSH, PRL, T, and E2 were measured with the ${ }^{125} \mathrm{I}-$ radioimmunoassay (GC-1500 Gamma radioimmunoassay counter, CHINA). Plasma glucose was measured using the glucose oxidase method (Beckman, USA) and plasma insulin using a chemiluminescence immunometric assay. Total cholesterol, triglyceride, HDL-C, LDL-C and UA were measured using an enzymatic calorimetric method (Beckman, USA).

\subsection{Statistical Analysis}

All statistical analysis were performed with Statistical Package for Social Science (SPSS) 13.0 for personal computers. Results were presented as means \pm SD if the distribution was normal. Comparisons between two groups were performed using Student-Newman-Keuls test (SNK) if measurement data were homoscedastic. Otherwise, comparisons were performed with Mann-Whitney U test. Correlation analysis between two groups was analyzed with Pearson analysis. P value $\leq 0.05$ was considered statistically significant, and two-tailed test was accepted.

\section{RESULTS}

\subsection{Comparison of Metabolic Features and Sex Hormones between Patients with PCOS and with T2DM}

Fifty women were diagnosed as PCOS as according to the Rotterdam criteria. Fifty-six patients were included in the newly-diagnostic Type 2 diabetes mellitus. The average age of the T2DM group was $43.3 \pm 12.6 \mathrm{yr}$. The average age of the PCOS group was $28.2 \pm 6.2$ yr. There was a big discrepancy in ages, yet most metabolic parameters were worse in the PCOS group (Table 1). The 
Table 1. Comparison of metabolic features in women with PCOS and with T2DM.

\begin{tabular}{ccc}
\hline & T2DM & PCOS \\
\hline BMI $\left(\mathrm{kg} / \mathrm{m}^{2}\right)$ & $24.8 \pm 3.4$ & $28.9 \pm 5.3^{* *}$ \\
WHR & $0.91 \pm 0.28$ & $0.98 \pm 0.34^{*}$ \\
SBP $(\mathrm{mmHg})$ & $157 \pm 33$ & $123 \pm 27^{*}$ \\
DBP $(\mathrm{mmHg})$ & $90 \pm 21$ & $83 \pm 17$ \\
TG $(\mathrm{mmol} / \mathrm{l})$ & $1.60 \pm 0.93$ & $2.22 \pm 2.06$ \\
CHOL $(\mathrm{mmol} / \mathrm{l})$ & $4.98 \pm 1.54$ & $4.27 \pm 0.79$ \\
HDL $(\mathrm{mmol} / \mathrm{l})$ & $1.09 \pm 0.52$ & $1.16 \pm 0.27$ \\
LDL $(\mathrm{mmol} / \mathrm{l})$ & $3.22 \pm 1.57$ & $2.42 \pm 0.83$ \\
UA (umol/l) & $258.2 \pm 89.46$ & $365.4 \pm 87.94^{* *}$ \\
FPG (mmol/l) & $9.81 \pm 3.29$ & $4.93 \pm 1.29^{* *}$ \\
2hPG (mmol/l) & $18.30 \pm 5.08$ & $8.22 \pm 3.10^{* *}$ \\
FINS (mmol/l) & $51.8 \pm 40.8$ & $127.8 \pm 76.9^{* *}$ \\
2h INS (mmol/l) & $295.1 \pm 193.7$ & $672.4 \pm 227.3^{* *}$ \\
HOMA-IR & $1.17 \pm 0.80$ & $2.20 \pm 1.54^{*}$ \\
\hline$*$
\end{tabular}

${ }^{*} \mathrm{p}<0.05,{ }^{* *} \mathrm{p}<0.01$ vs. T2DM. ${ }^{\triangle}$ have less clinical significance.

women with PCOS had higher BMI and WHR (average BMI: $28.9 \pm 5.3 \mathrm{~kg} / \mathrm{m}^{2}$; average WHR: $0.98 \pm 0.34$ ) than the women with T2DM (average BMI: $24.8 \pm 3.4 \mathrm{~kg} / \mathrm{m}^{2}$, $\mathrm{p}<0.01$; average WHR: $0.91 \pm 0.28, \mathrm{p}<0.05$ ). The plasma level of UA was significantly higher in the PCOS women $(365.4 \pm 87.94$ vs. $258.2 \pm 89.46 \mathrm{umol} / \mathrm{l}, \mathrm{p}<$ $0.001)$. These PCOS women had increased fasting (127.8 \pm 76.9 vs. $51.8 \pm 40.8 \mathrm{mmol} / \mathrm{l}, \mathrm{p}=0.001)$ and postprandial insulin levels $(672.4 \pm 227.3$ vs. $295.1 \pm 193.7$ $\mathrm{mmol} / \mathrm{l}, \mathrm{p}<0.001)$. The homeostasis model assessment index for insulin resistance was higher in PCOS women ( $2.20 \pm 1.54$ vs. $1.17 \pm 0.80, p=0.018)$. On the other hand, the new-diagnostic T2DM patients had higher CHOL, LDL-C and blood pressure than the PCOS patients (Table 1). Excluding the disturbance of age, only systolic blood pressure showed statistical significance (157 \pm 33 vs. $123 \pm 27 \mathrm{mmHg}, \mathrm{p}=0.03)$. The average fasting and postprandial plasma glucose was comparatively high in the T2DM group. But there was less clinical significance in the level of plasma glucose, because every patient with confirmed diagnosis of Type 2 diabetes was that of an inpatient with the purpose of controlling the high plasma glucose.

The ages of the patients with T2DM were between 31 and $55 \mathrm{yr}$, most patients with T2DM were menopausal. As a result, the levels of LH and FSH were significantly higher in T2DM women than in PCOS women (Table 2). The level of estrogen was low in T2DM (40.09 \pm 19.83 $\mathrm{pg} / \mathrm{ml}$ ). Although the women with PCOS were younger, the level of estrogen was even lower in the PCOS group than in the T2DM group ( $30.09 \pm 16.8 \mathrm{pg} / \mathrm{ml})$. The average testosterone was significantly increased in PCOS
Table 2. Comparison of hormonal features in women with PCOS and with T2DM.

\begin{tabular}{ccc}
\hline & T2DM & PCOS \\
\hline LH (mIU/ml) & $18.86 \pm 11.3$ & $10.71 \pm 4.96^{* *}$ \\
FSH $(\mathrm{mIU} / \mathrm{ml})$ & $28.83 \pm 18.1$ & $5.70 \pm 2.37^{* *}$ \\
LH/FSH & $0.65 \pm 0.52$ & $1.88 \pm 0.82^{*}$ \\
E2 (pg/ml) & $40.09 \pm 19.83$ & $30.09 \pm 16.84$ \\
PRL (ng/ml) & $20.58 \pm 30.31$ & $62.13 \pm 61.35^{* *}$ \\
T (ng/dl) & $0.154 \pm 0.12$ & $0.594 \pm 0.38^{* * *}$ \\
\hline${ }^{*}<0.05,{ }^{* *} \mathrm{p}<0.01$ vs. T2DM. & &
\end{tabular}

patients ( $0.594 \pm 0.38$ vs. $0.154 \pm 0.12 \mathrm{ng} / \mathrm{dl}, \mathrm{p}<0.01)$. The prolactin level was also significantly higher in women with PCOS than with T2DM $(62.13 \pm 61.35$ vs. $20.58 \pm 30.31 \mathrm{ng} / \mathrm{ml}, \mathrm{p}<0.01)$. The LH/FSH ratio in PCOS group was higher than in T2DM women $(1.88 \pm$ 0.82 vs. $0.65 \pm 0.52, \mathrm{P}=0.04$ ).

\subsection{LH after the GnRH Test in NGT IFG/IGTPcos and DMPcos}

After an OGTT test, patients with PCOS were divided into three groups, 16 patients in normal glucose tolerance group(NGT $\mathrm{PCOS}_{\mathrm{P}}$, 20 patients in impaired fasting glycaemia or impaired glucose tolerance((IFG/IGT $\left.\mathrm{PCOS}_{\mathrm{PC}}\right)$ group, and 14 patients in diabetes mellitus group $\left(\mathrm{DM}_{\mathrm{PCOS}}\right)$. The response of $\mathrm{LH}$ to the $\mathrm{GnRH}$ stimulation is significantly higher in $\mathrm{DM}_{\mathrm{PCO}}$ group than in $\mathrm{NGT}_{\mathrm{PCO}}$ group, especially at the 30 minutes mark (71.97 \pm 37.42 vs. $45.00 \pm$ $25.04 \mathrm{mIU} / \mathrm{ml}, \mathrm{p}<0.05)$ and at 120 minutes mark (69.53 \pm 43.23 vs. $36.72 \pm 17.47 \mathrm{mIU} / \mathrm{ml}, \mathrm{p}<0.05$ ) (Table 3).

\subsection{The Relationship between $\mathrm{LH}_{\text {peak }}$ in the GnRH Test and Metabolic Features}

$\mathrm{LH}_{\text {peak }}$ is positively correlated with fasting insulin $(\mathrm{P}=$ $0.004)$, postprandial insulin ( $P=0.012)$, HOMA-IR in$\operatorname{dex}(\mathrm{P}=0.018)$ and WHR $(\mathrm{P}=0.032)($ Table 4$)$.

\section{DISCUSSION}

Polycystic ovary syndrome is a common endocrine disorder in reproductive-aged women which is characterized by hyperandrogenism and chronic anovulation. It is likely that PCOS represents a complex inherited multifactorial trait, similarly to Type 2 diabetes. It was first reported in 1980 [5] and it was subsequently confirmed that women with PCOS were hyperinsulinemic, suggesting the presence of insulin resistance [6]. Insulin resistance is now recognized as a major risk factor for the development of Type 2 diabetes mellitus and it has been shown that polymorphism of insulin receptor substrate (IRS) is the common genetic foundation of the two diseases [7]. 
Table 3. LH after GnRH stimulation test in $\mathrm{NGT}_{\mathrm{PCOS}}$, IFG/IGT $\mathrm{PCOS}_{\mathrm{P}}$ and $\mathrm{DM}_{\mathrm{PCOS}}$.

\begin{tabular}{ccccccc}
\hline & $\mathrm{LH}_{0 \min }$ & $\mathrm{LH}_{15 \min }$ & $\mathrm{LH}_{30 \min }$ & $\mathrm{LH}_{60 \min }$ & $\mathrm{LH}_{90 \min }$ & $\mathrm{LH}_{120 \min }$ \\
\hline $\mathrm{NGT}_{\mathrm{PCOS}}$ & $9.48 \pm 2.96$ & $30.58 \pm 15.89$ & $45.00 \pm 25.04$ & $41.61 \pm 20.34$ & $38.37 \pm 18.15$ & $36.72 \pm 17.47$ \\
$\mathrm{IFG} / \mathrm{IGT}_{\mathrm{PCOS}}$ & $9.30 \pm 5.23$ & $40.72 \pm 33.37$ & $59.64 \pm 42.26$ & $50.43 \pm 38.48$ & $53.62 \pm 30.60$ & $58.42 \pm 22.95^{*}$ \\
$\mathrm{DM}_{\mathrm{PCOS}}$ & $9.39 \pm 4.82$ & $48.74 \pm 41.32$ & $71.97 \pm 37.42^{*}$ & $59.33 \pm 49.36$ & $60.14 \pm 42.07^{*}$ & $69.53 \pm 43.23^{*}$ \\
\hline
\end{tabular}

${ }^{*} \mathrm{p}<0.05$ vs. $\mathrm{NGT}_{\mathrm{PCOS}}$

Table 4. Correlation between $\mathrm{LH}_{\text {peak }}$ and metabolic parameters.

\begin{tabular}{ccccccccc}
\hline & BMI & SBP & DBP & Tg & TC & HDL & LDL \\
\hline Related Coefficient & 0.04 & 0.53 & 0.38 & 0.327 & 0.329 & -0.054 & 0.011 \\
P value & 0.884 & 0.052 & 0.163 & 0.276 & 0.272 & 0.854 & 0.969 \\
& WHR & UA & BG0h & BG2h & INS0h & INS2h & HOMA-IR \\
Related Coefficient & 0.612 & 0.177 & 0.097 & 0.155 & 0.732 & 0.674 & 0.725 \\
P value & 0.032 & 0.544 & 0.763 & 0.582 & 0.004 & 0.012 & 0.018 \\
\hline
\end{tabular}

Metabolic abnormalities occurring in both PCOS and T2DM have been separately widely studied. Although these two diseases have a more or less similar pathogenesis, there are, however, few studies that compare the metabolic disturbances of these two conditions. Our data showed that new-diagnostic PCOS women had higher BMI and WHR than the new-diagnostic T2DM patients. It might be an indication that PCOS women present with more severe insulin resistance than their T2DM counterparts. This was further underlined by the higher average HOMA-IR index in PCOS women. Besides the obvious abdominal obesity, they also had significantly higher uric acid. On the other hand, not all metabolic parameters in the PCOS group were inferior to those in the T2DM group. Our study showed the average systolic blood pressure, fasting plasma glucose and postprandial plasma glucose were all significantly higher in the T2DM group.

It is known that with the GnRH stimulation test, LH reaction will be exaggerated in PCOS women. The results of our study further confirmed this notion. Furthermore, our study also suggested the LH reaction in PCOS women with glucose abnormality will be much higher than those with normal glucose tolerance. The peak value of LH after GnRH test is positively correlated with insulin levels, WHR and HOMA-IR as according to the Pearson analysis. In other words, insulin resistance disturbs the function of the thalamus-pituitary-gonad axis.

Insulin resistance in PCOS can be secondary to a postbinding defect in insulin receptor signaling pathways, and elevated insulin levels may have gonadotropinaugmenting effects on ovarian function. The excessive insulin binding to the insulin receptors beside the anterior pituitary stimulates release of luteinizing hormone. A proposed mechanism for anovulation and elevated androgen levels suggests that under the increased stimula- tory effect of LH, stimulation of the ovarian theca cells is increased. In turn, these cells increase the production of androgens (e.g. testosterone, androstenedione). The excessive insulin can also directly enhance the activity of $17 \alpha$-hydroxylase in theca cells. As a result, androgens production is increased. The excessive insulin inhibits the normal synthesis of sex-hormone binding globulin, than to enhance the level of free testosterone. Hyperandrogenaemia obstructs the normal development of ovary and causes the polycystic ovaries. Hyperandrogenaemia inversely aggravates the metabolic disturbances [8]. Insulin resistance induces a vicious circle of hyperandrogenaemia and metabolic disturbances. It has been reported that drugs increasing insulin sensitivity can not only improve metabolic status but also can induce ovulation in women with PCOS [9].

The main limitation of this study would be that the patients recruited were of different age groups, however, this discrepancy should not prevent us from analyzing and differentiating between these two diseases that have a lot in common when it comes to hormonal and endocrinological changes. A much larger study of metabolic disorders should be undertaken on patients of the similar age groups so as to obtain more accurate results.

\section{CONCLUSION}

Women with PCOS have more severe metabolic disturbance than women with T2DM except for systolic blood pressure and plasma glucose. The abnormal glucose tolerance indicates the higher peak of $\mathrm{LH}$ in GnRH stimulation test. Insulin resistance affects the function of the thalamus-pituitary-gonad axis.

\section{ACKNOWLEDGEMENTS}

The work was supported by Foundation of Shanghai First People’s 
Hospital (09B03). This study was supported by National Natural Science Foundation of China (Grant No. 81100616 and Grand No. 81000333).

\section{COMPETING INTERESTS}

The authors declare that they have no competing interests.

\section{REFERENCES}

[1] Salley, K.E., Wickham, E.P., Cheang, K.I., Essah, P.A., Karjane, N.W. and Nestler, J.E. (2007) Glucose intolerance in polycystic ovary syndrome-A position statement of the Androgen Excess Society. The Journal of Clinical Endocrinology and Metabolism, 92, 4546-4556. http://dx.doi.org/10.1210/jc.2007-1549

[2] Moran, L.J., Misso, M.L., Wild, R.A., Norman, R.J. (2010) Impaired glucose tolerance, Type 2 diabetes and metabolic syndrome in polycystic ovary syndrome: A systematic review and meta-analysis. Hum Reprod Update, 16, 347363. http://dx.doi.org/10.1093/humupd/dmq001

[3] Wild, R.A., Carmina, E., Diamanti-Kandarakis, E., Dokras, A., Escobar-Morreale, H.F., Futterweit, W., Lobo, R., Norman, R.J., Talbott, E. and Dumesic, D.A. (2010) Assessment of cardiovascular risk and prevention of cardiovascular disease in women with the polycystic ovary syndrome: A consensus statement by the Androgen Excess and Polycystic Ovary Syndrome (AE-PCOS) Society. The Journal of Clinical Endocrinology and Metabolism, 95, 2038-2049. http://dx.doi.org/10.1210/jc.2009-2724
[4] Levy, J.C., Matthews, D.R. and Hermans, M.P. (1998) Correct homeostasis model assessment (HOMA) evaluation uses the computer program[J]. Diabetes Care, 21, 2191-2192. http://dx.doi.org/10.2337/diacare.21.12.2191

[5] Burghen, G.A., Givens, J.R. and Kitabchi, A.E. (1980) Correlation of hyperandrogenism with hyperinsulinism in polycystic ovarian disease. The Journal of Clinical Endocrinology and Metabolism, 50,113-116. http://dx.doi.org/10.1210/jcem-50-1-113

[6] Dunaif, A. and Thomas, A. (2001) Current Concepts in the polycystic ovary syndrome. Annual Review of Medicine, 52, 401-404. http://dx.doi.org/10.1146/annurev.med.52.1.401

[7] Lin, T.C., Yen, J.M., Gong, K.B., et al. (2006) Abnormal glucose tolerance and insulin resistance in polycystic ovary syndrome amongst the Taiwanese population- not correlated with insulin receptor substrate-1 Gly972Arg/ Ala513Pro polymorphism. BMC Medical Genetics, 7, 3638. http://dx.doi.org/10.1186/1471-2350-7-36

[8] Fruzzetti, F., Perini, D., Lazzarini, V., et al. (2009) Hyperandrogenemia influences the prevalence of the metabolic syndrome abnormalities in adolescents with the polycystic ovary syndrome. Gynecological Endocrinology, 25, 335-343. http://dx.doi.org/10.1080/09513590802630146

[9] Ghazeeri, G., Kutteh, W.H., Bryer-Ash, M., et al. (2003) Effect of rosiglitazone on spontaneous and clomiphene citrate-induced ovulation in women with polycystic ovary syndrome. Fertility and Sterility, 79, 562-566. http://dx.doi.org/10.1016/S0015-0282(02)04843-4 